\title{
Colorful wall-bricks with superhydrophobic surfaces for enhanced smart indoor humidity control
}

Xiaopeng Liu, ${ }^{\mathrm{a}}$ Zhang Chen, ${ }^{\mathrm{a}^{*}}$ Guang Yang, ${ }^{\mathrm{a}}$ zong tao Zhang, ${ }^{\mathrm{b*}}$ Yanfeng Gao ${ }^{\mathrm{a}^{*}}$

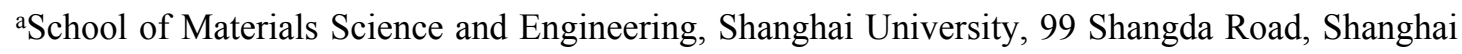
200444, China

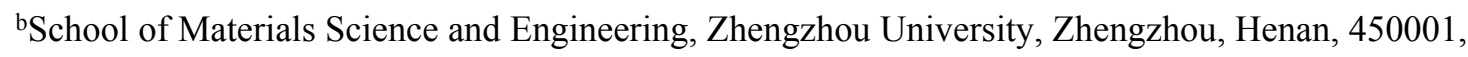
China

Email: yfgao@shu.edu.cn; chenzhang@shu.edu.cn; ztzhang@zzu.edu.cn.

This supporting information consists of 11 figures, 2 tables and 4 movies. 

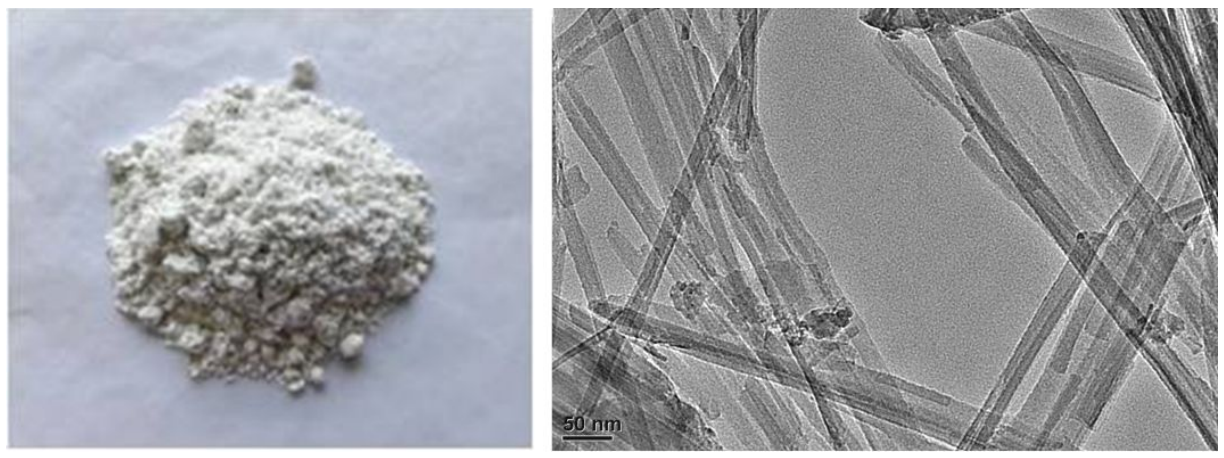

Figure S1. Digital and TEM images of the sepiolite.
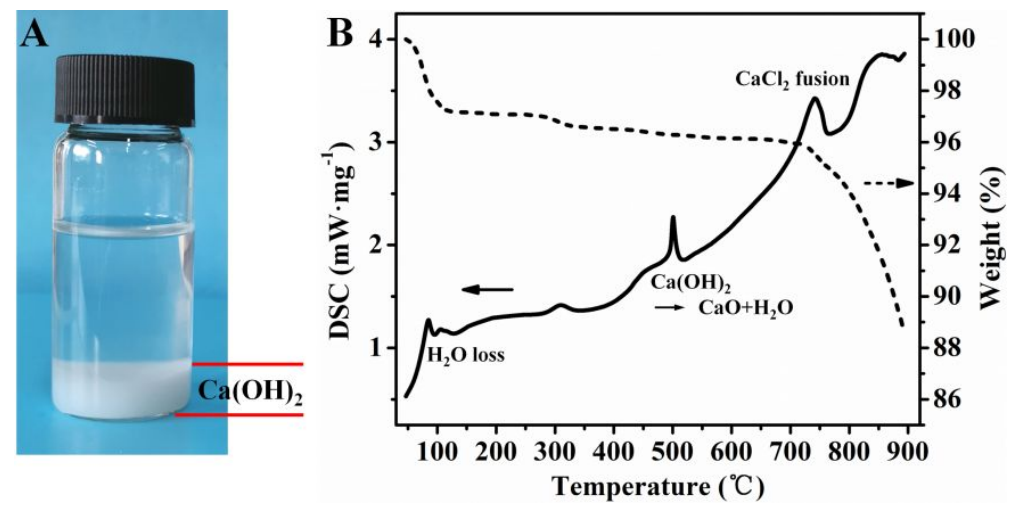

Figure S2. (A) Image and (B) DSC-TG curves of the filtrate of acid activated sepiolite with pH10.

The three main peaks are water loss, the decomposition of the slaked lime and $\mathrm{CaCl}_{2}$ fusion.
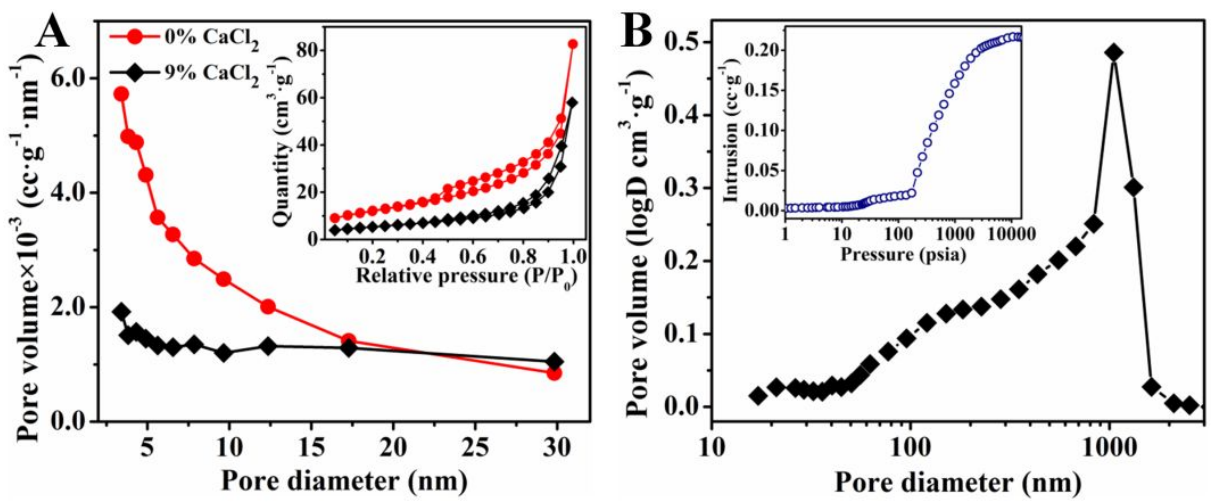

Figure S3. (A) Barrett-Joyner-Halenda pore size distributions and the embedded nitrogen

adsorption and desorption isotherms. (B) Macropores distribution and the embedded mercury cumulative intrusion curve. 


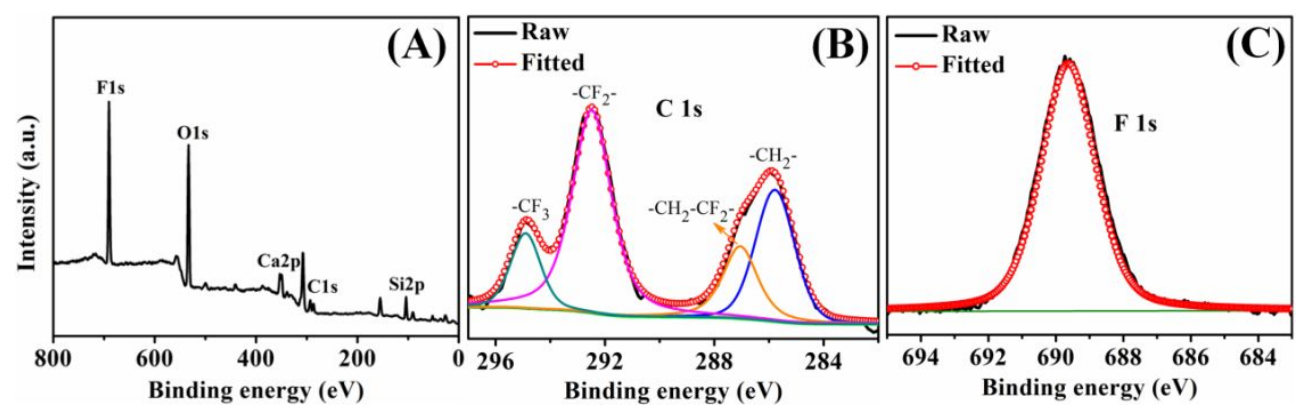

Figure S4. XPS patterns of wall-brick. (A) Full range XPS spectra; (B) the high-resolution C spectrum; (C) the high-resolution F spectrum.

We further use Cassie-Baxter model to estimate the apparent CA as follows: ${ }^{1-3,22}$

$$
\cos \theta_{C A}=-1+\frac{S_{c}}{S_{f l a t}} \cdot\left(1+\cos \theta_{f l a t}\right)
$$

where $\mathrm{S}_{\mathrm{c}}$ and $\mathrm{S}_{\text {flat }}$ are the surface area that is in contact with the liquid. $\theta_{\text {flat }}$ is the contact angle of a flat surface. In this discussion, the flat surface is deemed to a microscopic surface on the micro-structure surface, which typically exists in the wall-brick without $\mathrm{CaCl}_{2}$ (Figure 2A). Image $\mathrm{J}$ software was used to estimate the contact surface area of Figure 2B. The CA calculated according to the model is 154.2 $2^{\circ}$, which is comparable to the measured CA in Table 1.

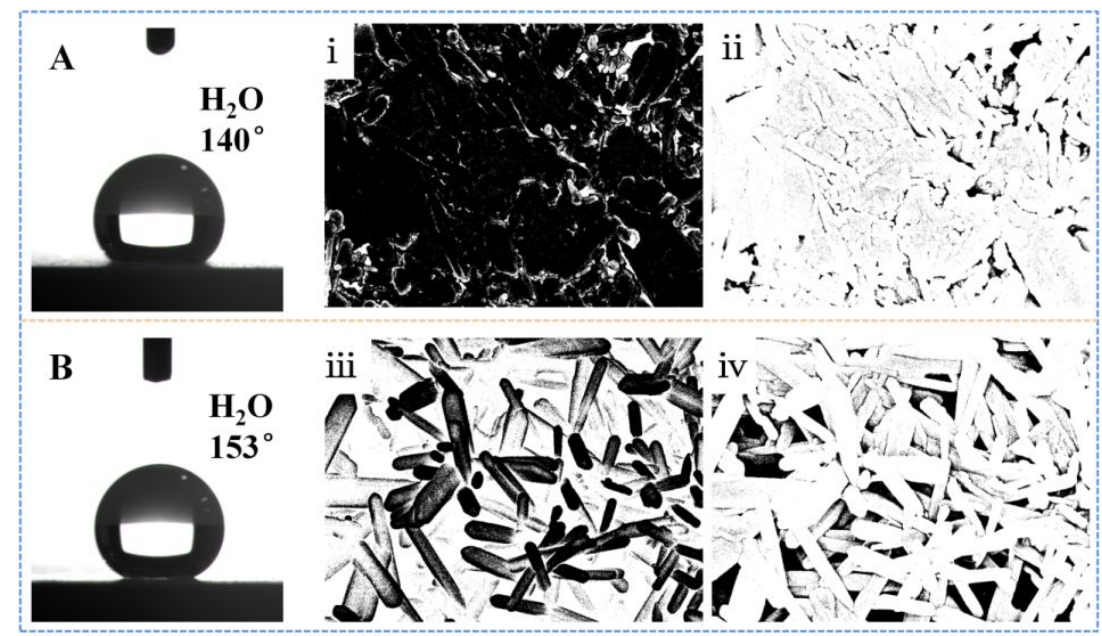

Figure S5. Contact angle (CA) of $5 \mu \mathrm{L}$ water droplet on the surface of the wall-brick (A) without 
$\mathrm{CaCl}_{2}$, (B) with $\mathrm{CaCl}_{2}$. The calculation of contact surface area by Image $\mathrm{J}$ software: ( $\mathrm{i}$ ) (iii) the sepiolite fibres with deep gray are in contact with the liquid $\mathrm{S}_{\mathrm{c}}$, ( ii ) (iv) the deep gray area is air pockets. Original morphology image is Figure 2A, 2B.
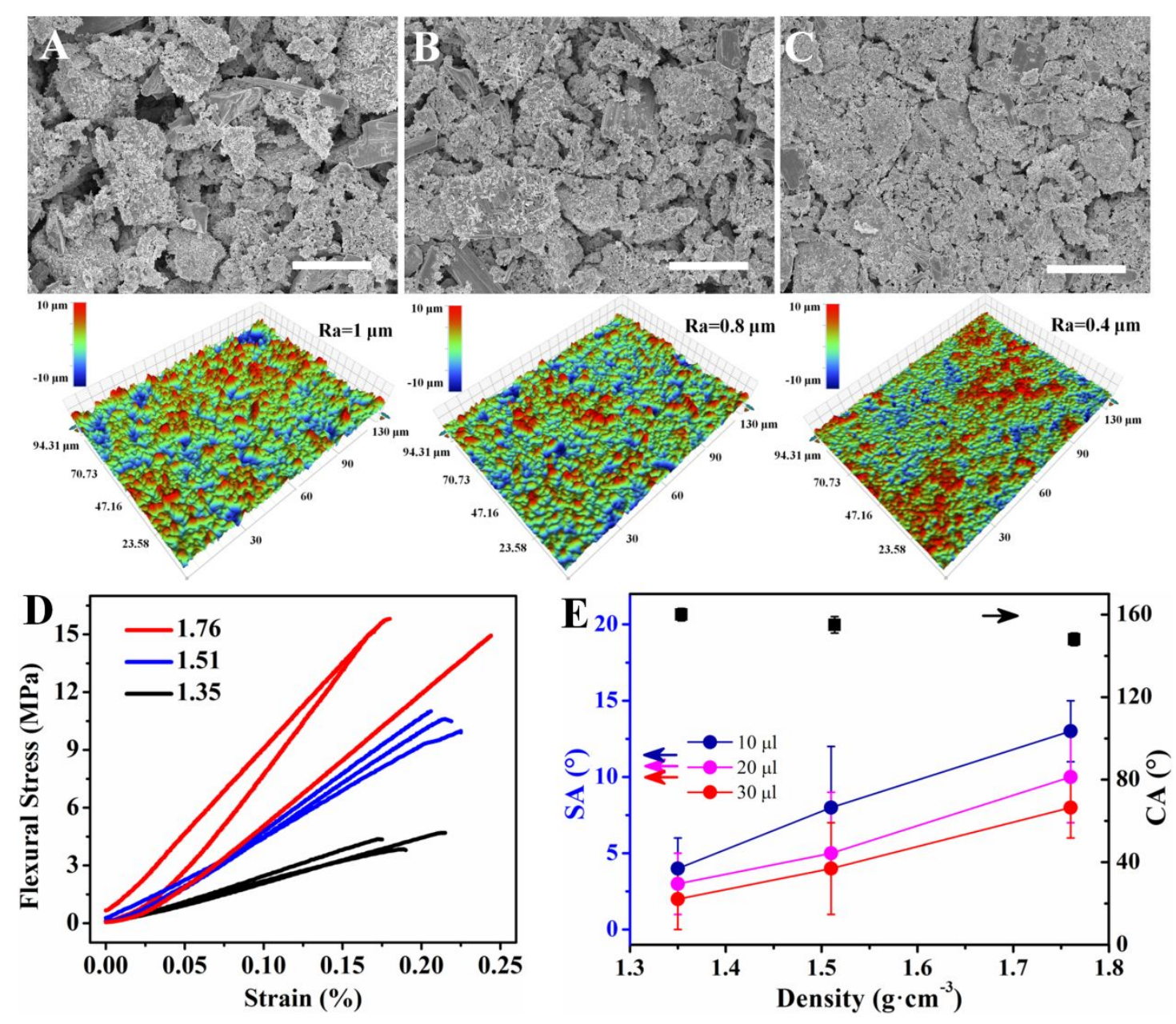

Figure S6. SEM images and the corresponding Ra values of wall-brick with the density of (A)

$1.35 \mathrm{~g} \cdot \mathrm{cm}^{-3}$, (B) $1.51 \mathrm{~g} \cdot \mathrm{cm}^{-3}$, (C) $1.76 \mathrm{~g} \cdot \mathrm{cm}^{-3}$, (D) stress-strain curves in 3-point bending for wall-brick with the density of $1.35,1.51,1.76 \mathrm{~g} \cdot \mathrm{cm}^{-3}$. Three specimens were tested for each density. (E) SA for different water droplet volume as a function of brick density. With the increasing of wall-brick density, SA increases and CA decreases. 

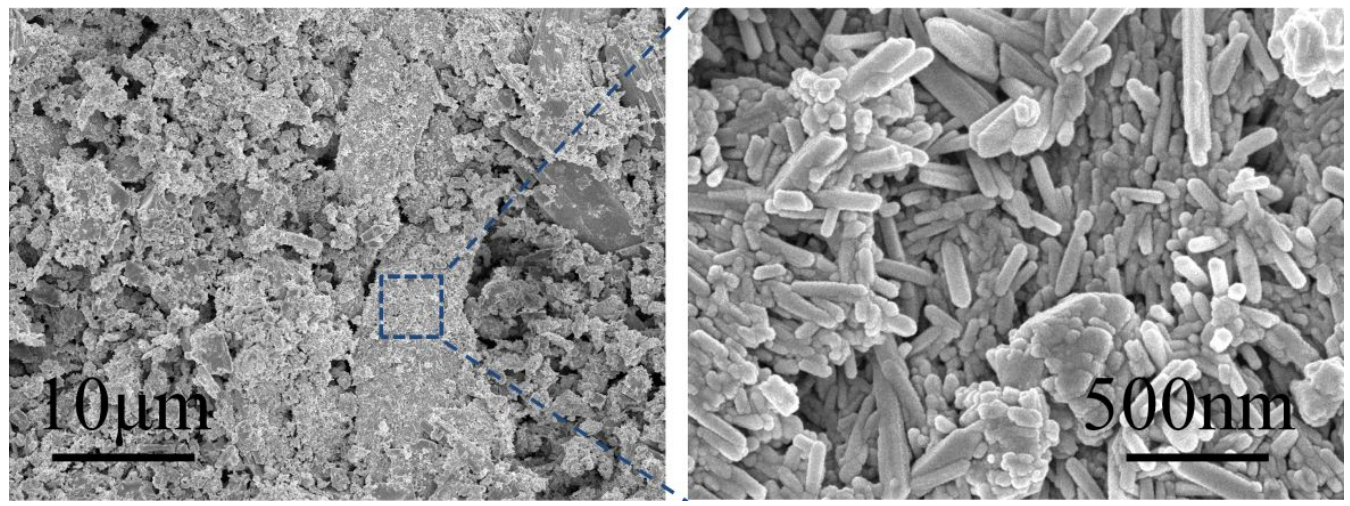

Figure S7. SEM surface morphologies of wall-brick after 100 wipes.

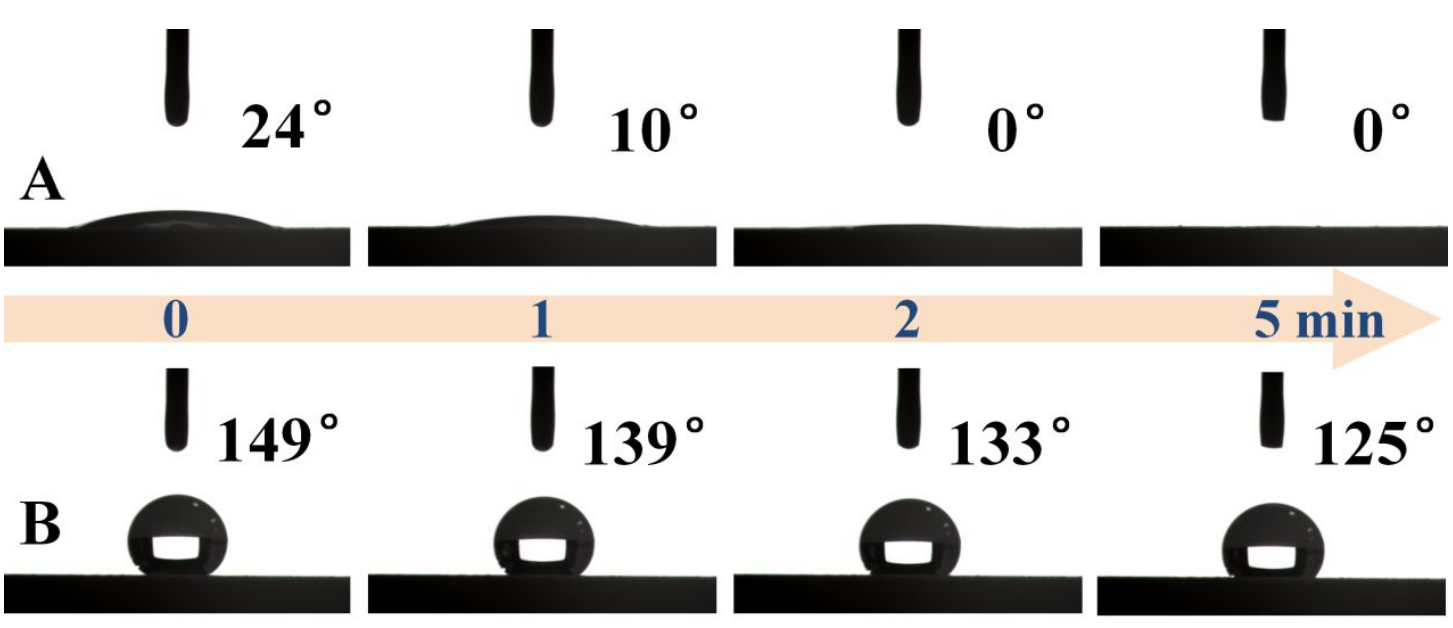

Figure S8. Contact angle (CA) of $5 \mu \mathrm{L}$ colza oil after $0,1,2,5 \mathrm{~min}$ on the wall-brick, (A) before and (B) after hydrophobic modification.
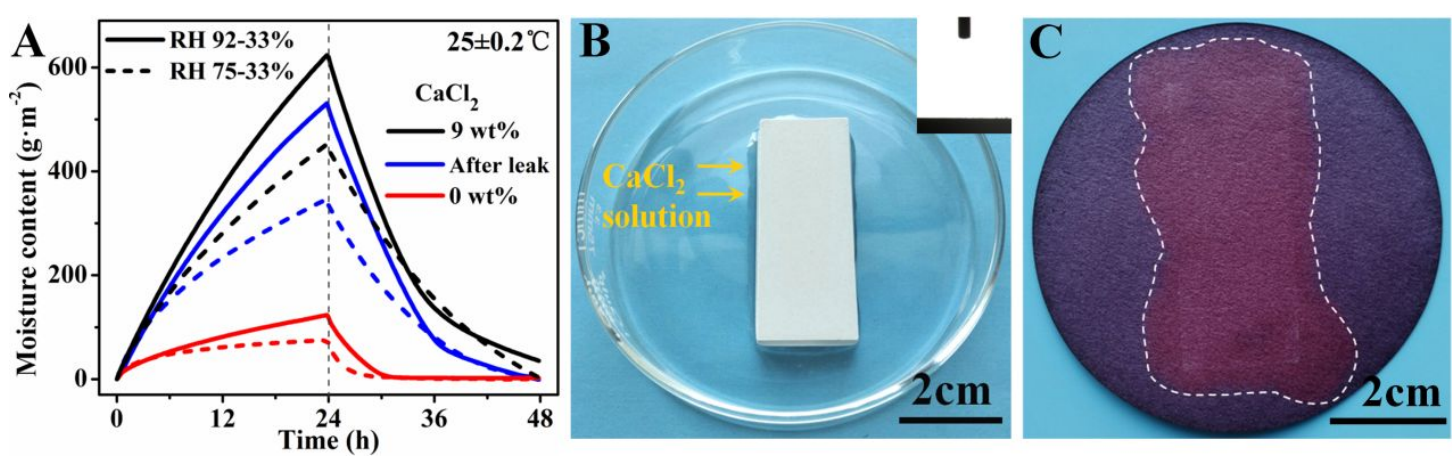

Figure S9. (A) The moisture adsorption-desorption contents of pristine hydrophilic wall-brick with or without $\mathrm{CaCl}_{2}$. (B) Digital images of the high humidity leakage test for pristine 
hydrophilic wall-brick with $9 \mathrm{wt} \% \mathrm{CaCl}_{2}$. (C) The corresponding leakage trace on litmus papers.

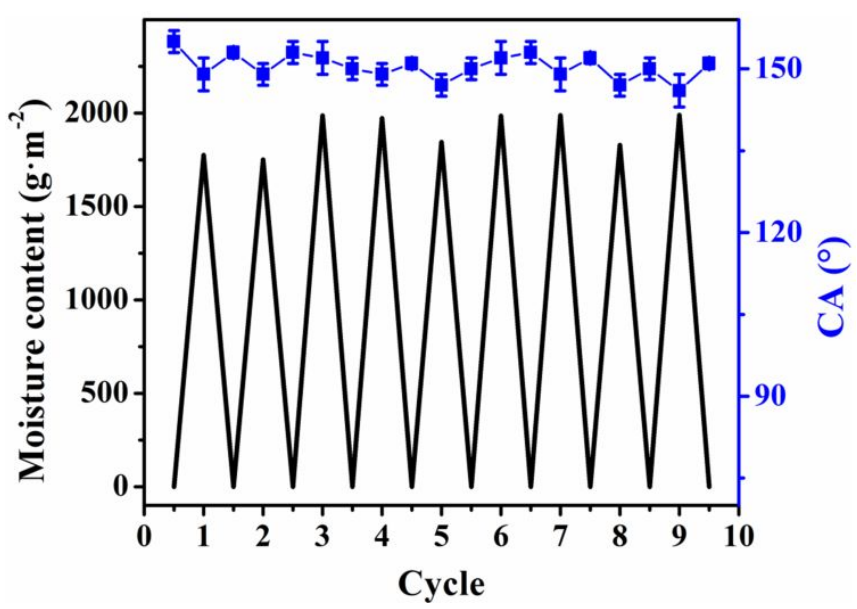

Figure S10. 9 cycles for the 4 days moisture adsorption-desorption and the corresponding CA for both saturated water status and dehydrated status.
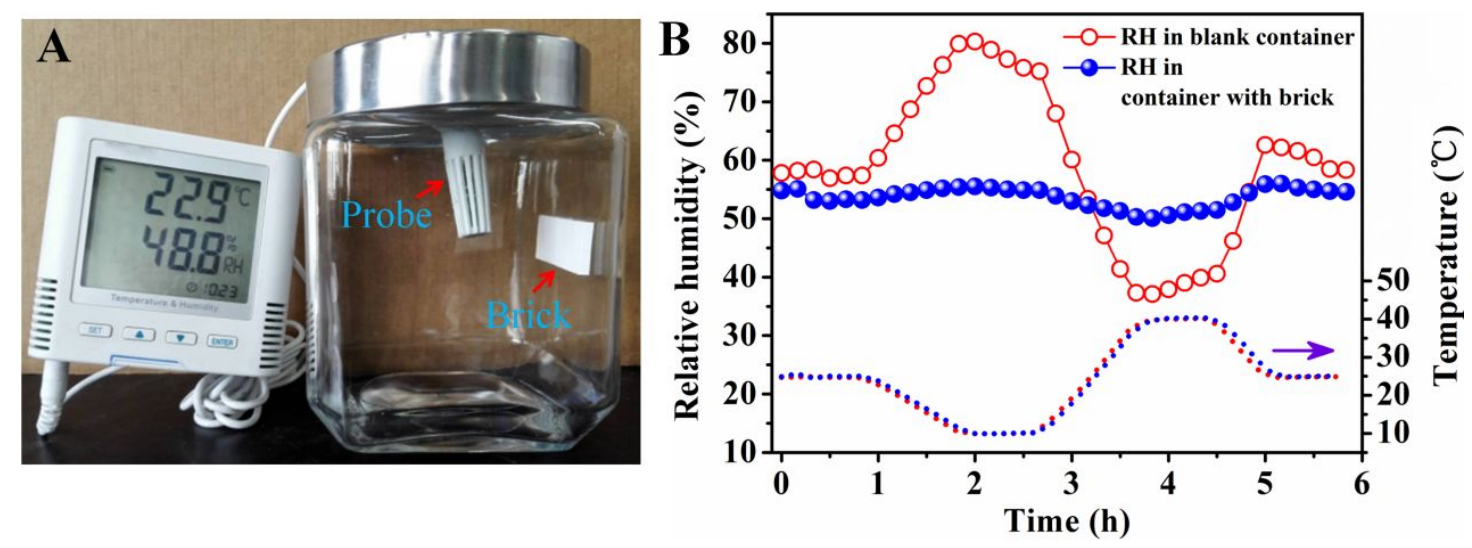

Figure S11. (A) Test apparatus and (B) curves of the humidity control property of wall-brick (size:

$20 \times 50 \times 4.5 \mathrm{~mm}$ ) in an airtight vessel (volume: $0.8 \mathrm{~L}$ ) at various temperatures. 
Table S1. Compositions of wall-bricks before/after sintering and low-melting glass.

\begin{tabular}{ccccc}
\hline Composition (wt.\%) & green specimen & with $\mathrm{CaCl}_{2}$ & without $\mathrm{CaCl}_{2}$ & Low-melting glass \\
\hline $\mathrm{SiO}_{2}$ & 46.9 & 50.2 & 52.8 & 37.3 \\
$\mathrm{MgO}$ & 17.6 & 20.7 & 23.6 & 20.4 \\
$\mathrm{CaO}$ & 15.5 & 15.7 & 16.2 & 3.7 \\
$\mathrm{CaCl}_{2}$ & 16.8 & 9.1 & 0.2 & - \\
$\mathrm{Na}_{2} \mathrm{O}$ & 0.7 & 1.8 & 2.2 & 19.8 \\
$\mathrm{Al}_{2} \mathrm{O}_{3}$ & 0.5 & 0.9 & 0.7 & 3.8 \\
$\mathrm{ZnO}$ & - & 0.8 & 0.7 & 7.2 \\
$\mathrm{P}_{2} \mathrm{O}_{5}$ & - & 0.3 & 0.5 & 4.0 \\
Ignition loss & 2.0 & 0.5 & 3.1 & 3.8 \\
\hline
\end{tabular}

Table S2. Physical properties and contact angle of $\mathrm{CaCl}_{2}$ solution at $25^{\circ} \mathrm{C}$.

\begin{tabular}{cccccc}
\hline $\mathrm{CaCl}_{2}$ solution & Purified water & $15 \mathrm{wt} \%$ & $23 \mathrm{wt} \%$ & $30 \mathrm{wt} \%$ & $38 \mathrm{wt} \%$ \\
\hline Saturated vapor pressure $(\mathrm{kPa})$ & 3.169 & 3.011 & 2.852 & 2.218 & 1.585 \\
Surface tension $\left(\mathrm{mN} \cdot \mathrm{m}^{-1}\right)$ & 72.8 & 74.9 & 85.4 & 97.3 & 103.5 \\
Molar volume $\left(\times 10^{-6} \mathrm{~m}^{3} \cdot \mathrm{mol}^{-1}\right)$ & 18.1 & 30.1 & 32.6 & 34.9 & 39.5 \\
Contact angle $\left({ }^{\circ}\right)$ & $154 \pm 2$ & $157 \pm 3$ & $158 \pm 2$ & $>160$ & $>160$ \\
\hline
\end{tabular}

\section{References}

(1) Liu, H.; Wang, Y. D.; Huang, J. Y.; Chen, Z.; Chen, G. Q.; Lai, Y. K. Bioinspired surfaces with superamphiphobic properties: concepts, synthesis, and applications. Adv. Funct. Mater. $2018,28,1707415$.

(2) Wu, Y.; Zeng, J.; Si, Y. S.; Chen, M.; Wu, L. M. Large-Area preparation of robust and transparent superomniphobic polymer films. ACS Nano 2018, 12, 10338-10346.

(3) Choi, W.; Tuteja, A.; Chhatre, S.; Mabry, J. M.; Cohen, R. E.; McKinley, G. H. Fabrics with tunable oleophobicity. Adv. Mater. 2009, 21, 2190-2195. 